\title{
Systemic delivery of synthetic microRNA-451 is an effective therapeutic strategy for the treatment of lung cancer
}

\author{
BAISONG LOU and XIN ZHOU \\ Department of Medical Oncology, The First Hospital of Qiqihaer City of Heilongjiang, \\ Qiqihaer, Heilongjiang 161005, P.R. China
}

Received June 17, 2014; Accepted March 2, 2015

DOI: $10.3892 / \mathrm{ijmm} .2015 .2147$

\begin{abstract}
MicroRNAs (miRNAs) can function as tumor suppressors and may provide an efficient strategy for the eradication of cancer. Specific miRNAs can be reintroduced into tumor cells to complement loss of tumor suppression activities. The aim of the present study was to develop miRNA delivery formulation using synthesized miR-451. miR-451 was detected using RT-PCR and tissues were analyzed using immunohistochemical analysis. miRNA delivery formulation proved to be effective either locally or systemically. miR-451 accumulation became evident in tumor cells and exerted an anti-proliferative function. The intravenous delivery of formulated miR-451 did not induce any deregulation in the cytokine levels and liver enzymes. Taken together, the results provide insight into the concept of the systemic delivery of synthetic mimics for tumor suppressor miR-451 and provide potential implications for miRNA therapy in clinical practice.
\end{abstract}

\section{Introduction}

MicroRNAs (miRNAs or miRs) are small non-coding RNA molecules that can function as a post-transcriptional factor for gene expression and determine cell fate by modulating various cell pathways. miRNAs may exert their functions by base pairing with the 3'-untranslated region (3'-UTR) of target mRNAs (1). Deregulated miRNAs are often biomarkers of cellular events that contribute to the onset of malignancy such as tumor (2). In vitro and in vivo studies have identified critical roles for miRNAs in the regulation of tumor cell invasion, metastasis and migration (3). One potential reason for miRNAs being downregulated in many cancer cells is that miRNAs may target various oncogenic signaling pathways can serve as tumor suppressors. Downregulation of tumor-suppressor miRNAs in various tumor tissues has been reported while

Correspondence to: Dr Baisong Lou, Department of Medical Oncology, The First Hospital of Qiqihaer City of Heilongjiang, 20 Park Road, Longsha, Qiqihaer, Heilongjiang 161005, P.R. China E-mail: manuloubaisong@163.com

Key words: microRNA-451, lung cancer treatment, apoptosis, systemic delivery of those the reduction of miR-451 expression has also been extensively shown (4-7). The $m i R-451$ gene is located on chromosome 17q11.2 and is associated with the occurrence of many tumor types (8). It was reported that miR-451 may exert a tumor-suppressor function by targeting MDF1/P-protein expression in human cancer cell lines. In particular, the tumor suppressive potential of miR-451 is also evident in lung cancer cells by targeting Ras-related protein 14 (RAB14). miR-451 can be transcriptionally induced or repressed by many transcription factors. For example, SMAD can transcriptionally upregulate miR-451 expression while the signal transducer and activator of transcription 3 (STAT3) may play an opposite role $(9,10)$. Overexpression of miR-451 inhibits the growth and the metastatic potential of cultured cancer cells $(8,11,12)$. Therefore, miR-451 may play an anti-proliferative role in tumor cells.

Given the anti-proliferative function of miR-451, it may be potentially applied in cancer treatment. However, there is currently no direct in vivo evidence that miR-451 serves as a therapeutic agent. In the present study, we showed that miR-451 can exert its tumor suppressor activity in vivo. Local treatment and intravenous injection of miR-451 significantly reduced tumor malignancy as evaluated by immunohistochemical staining. Furthermore, systemic delivery of formulated miR-451 proved to be a safe strategy as demonstrated by measuring the serum levels of multiple cytokines under different treatment conditions. Therefore, the present results may provide insight into restoring normal functionality in cancer cells via miRNA delivery.

\section{Materials and methods}

Human tissue samples, cell lines and oligos. Sixteen flashfrozen NSCLC tumor samples and corresponding normal adjacent tissues (NAT) were obtained from The First Hospital of Qiqihaer City. Five formalin-fixed paraffin-embedded (FFPE) lung tumor samples were generously supplied by Nanjing Gulou Hospital. The A549, H460, H1299, H226, H522, H596, SW-900, Wi-38 and CALU-3 lung cancer cell lines were purchased from the Shanghai Institutes for Biological Sciences, Chinese Academy of Sciences (Shanghai, China) and cultured according to the instructions. Transfection was evaluated as described previously (13). Proliferation was assessed by Alamar Blue (Invitrogen, Carlsbad, CA, USA) 4-6 days after transfection. Cells $\left(4 \times 10^{6}\right)$ were electroporated 
with $2 \mu \mathrm{mol} / 1 \mathrm{miRNA}$ in $100 \mu \mathrm{l}$ Opti-MEM (Sigma, St. Louis, MO, USA) using colony formation assays. Cells $\left(2 \times 10^{3}\right)$ were seeded in 100-mm dishes. After 30 days, the colonies were stained with $2 \%$ crystal violet and colonies containing $>50$ cells were counted. Synthetic miRNAs and siRNAs were purchased from Applied Biosystems (Foster City, CA, USA) and Dharmacon (Lafayette, CO, USA).

Xenografts for lung cancer. The animal experiments were performed according to prescribed protocols and approved by the Ethics Committee of the First Hospital of Qiqihaer City. H460 cells were collected and mixed with Matrigel (Sigma) at a 1:1 ratio by volume. Cells $\left(4 \times 10^{6}\right)$ in $100 \mu 1$ of Matrigel solution were injected in the back region of female non-obese diabetic/severe combined immunodeficient mice (NOD/SCID; Model Animal Research Center, Nanjing, China). miRNA were formulated using a MaxSuppressor in vivo RNA-Lancer II (Bioo Scientific, Inc., Austin, TX, USA). Formulated miRNAs were administered intratumorally (i.t.) or intravenously (i.v.) by tail vein injections when the tumor size was $>150 \mathrm{~mm}^{3}$. The tumors were collected, split and placed in $15 \%$ formalin for histology.

$R T-P C R$. RNA isolation from cells or tissue samples was performed with the mirVana miRNA Isolation kit (Ambion, Austin, TX, USA) according to the manufacturer's instructions. For RT-PCR detection of miR-451 oligonucleotide, mature miR-451 was reverse-transcribed with specific RT primers quantified with specific TaqMan probes: 5'-CTC AACTGGTGTCGTGGAGTCGGCAATTCAGTTGAGAAA CTCAG-3'. The raw data were normalized by $U 6$ small nuclear RNA using TaqMan miRNA assays (Applied Biosystems).

Histology and immunohistochemistry. Tumor tissues were fixed in formalin and embedded in paraffin using the Microm Tissue Embedding Center (Labequíp, Ltd., Markham, ON, Canada). The secretions were cut $(5 \mu \mathrm{m})$ and stained with hematoxylin and eosin (H\&E). For immunohistochemical staining, the sections were hydrated and blocked with $4 \%$ $\mathrm{H}_{2} \mathrm{O}_{2}$ in water for $15 \mathrm{~min}$. Antigen retrieval was performed with $20 \mathrm{mmol} / \mathrm{l}$ citrate buffer ( $\mathrm{pH} 6.0$ ) for $15 \mathrm{~min}$ followed by cooling for 20 min and washing in TBS with Tween-20 [TTBS: $50 \mathrm{mmol} / 1$ Tris- $\mathrm{HCl}(\mathrm{pH} 7.4), 150 \mathrm{mmol} / \mathrm{l} \mathrm{NaCl}$, $0,1 \%$ Tween-20]. The slides were treated with BioCare blocking reagent (BioCare Medical, Concord, CA, USA) for 15 min to block non-specific binding. The slides were then incubated with primary antibodies against caspases-3 (Sigma) for $40 \mathrm{~min}$ and subsequently washed in TTBS for $30 \mathrm{~min}$ at $20^{\circ} \mathrm{C}$. After washing, the slides were incubated with anti-goat horseradish peroxidase-conjugated secondary antibodies (BioGenex, Fremont, CA, USA) for $30 \mathrm{~min}$ at $20^{\circ} \mathrm{C}$. The slides were then counterstained with hematoxylin.

Cytokine measurement and blood chemistries. Female BALB/c mice (Model Animal Research Center, Nanjing, China) were injected i.v. in a lateral tail vein with $150 \mu \mathrm{g}$ of miRNA formulated with MaxSuppressor in vivo RNA-Lancer II. The mice were anesthetized with isoflurane. Blood was collected through cardiac puncture at selected time points after injection into a serum separator tube for isolation or EDTA tube for chemical

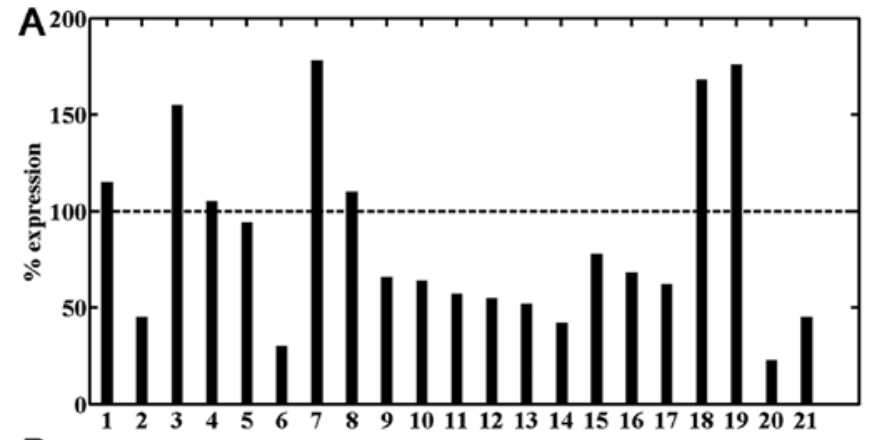

B

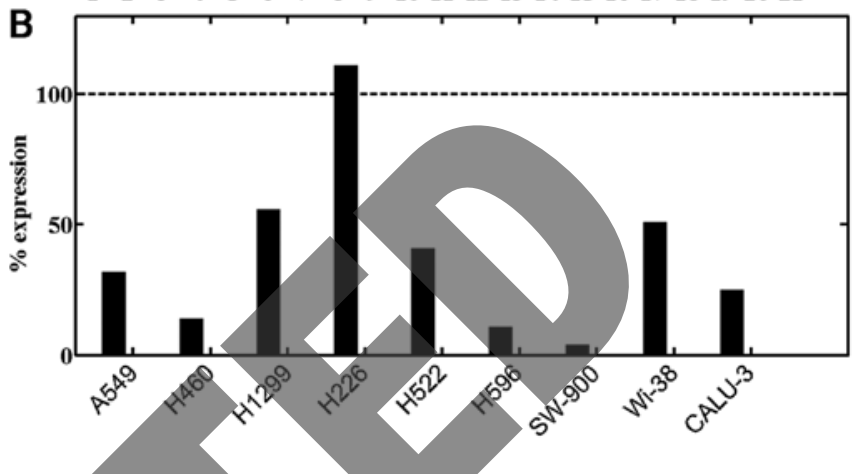

Figure 1. miR-451 expression in human NSCLC. (A) RT-PCR analysis of miR-451 in 21 NSCLC tumor samples [normal adjacent tissues (NATs) included and (B) various NSCLC cell lines. Raw Ct values were normalized as described in Materials and methods. The data were expressed as percent expression compared with corresponding NATs. Samples 1-16, flash-frozen; samples 17-21, formalin-fixed paraffin-embedded (FFPE). Dashed lines are guidelines for $100 \%$

analysis. Cytokine levels were determined using a Fluorokine MultiAnalyte Profiling kit (R\&D Systems, Minneapolis, MN, USA) and the Luminex 100 IS instrument. Blood chemistry was conducted at Nanjing Gulou Hospital.

\section{Results}

miR-451 is frequently downregulated in tumor cells. To identify whether the levels of miR-451 is altered in NSCLC patients, we tested 21 human tumor samples. miR-451 expression was evaluated by RT-PCR and NATs and tumor samples were compared. The results showed that of the 21 samples, 14 samples exhibited reduced miR-451 expression (Fig. 1A). Additionally, miR-451 was frequently downregulated in well-established tumor cell lines (Fig. 1B). Notably, for the upregulation of miR-451 in H266 cells, the level of miR-451 was not elevated substantially (Fig. 1B). These results suggested that miR-451 is frequently suppressed in human tumor cells.

miR-451 inhibits the growth of cancer cells. The abovementioned results suggested a malignant phenotype when miR-451 was downregulated. We determined whether miR-451 may interfere with lung cancer cell proliferation. We transiently transfected NSCLC cells of various types with miR-451 and measured the proliferation status at $48 \mathrm{~h}$ post-infection. Negative control (miR-NC), which does not target a specific human gene product was also transfected separately. The results suggested that the effect of growth inhibition was evident among the various tumor cell lines (Fig. 2A). Notably, 
A

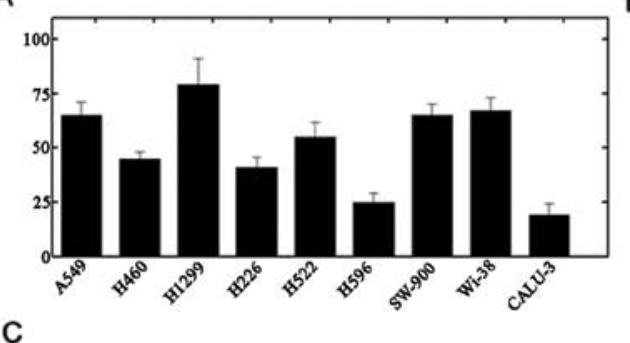

C

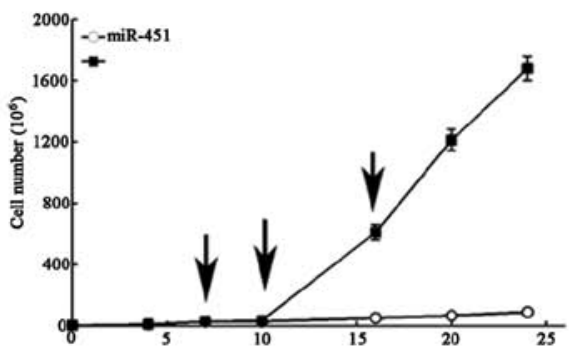

B

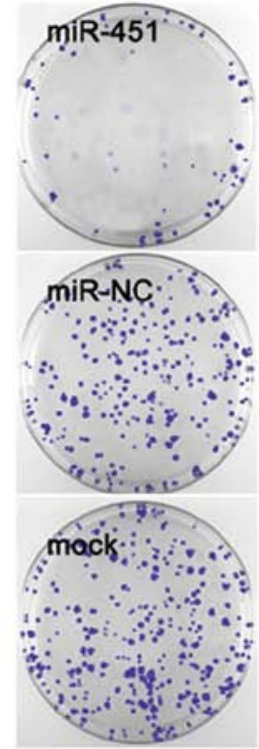

D
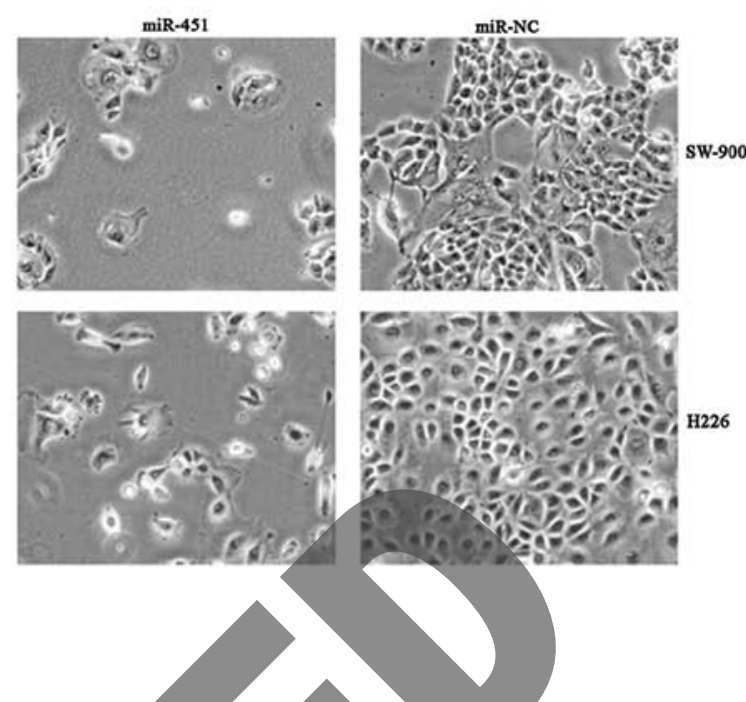

Figure 2. miR-451 inhibits activities of lung cancer cells. (A) Cancer cells were transfected with $50 \mathrm{nmol} / 1 \mathrm{miR}-451$ or miR-NC. Data are presented in three replicates. Bars, standard deviation (SD). (B) Colony formation assay using H1299 cells. Cells were transfected with miR-451, miR-NC and seeded at $3 \times 10^{3}$ cells $/ 100-\mathrm{mm}$ dish. After 30 days, cells were stained with $2 \%$ crystal violet. Colonies containing $>50$ cells were counted. (C) Long term effect of miR-451. $\mathrm{H} 226$ cells were transfected in triplicate with miR-451 and miR-NC, seeded, and propagated in regular growth medium. When the control cells reached confluence, the cells were collected, counted, and transfected again with the respective miRNAs. Data show three replicates. Arrows, transfection events. (D) Cellular morphology of SW-900 and H226 lung cancer cells 48 h post-transfection with miR-451 or miR-NC. Micrographs were taken using the x10 objective.

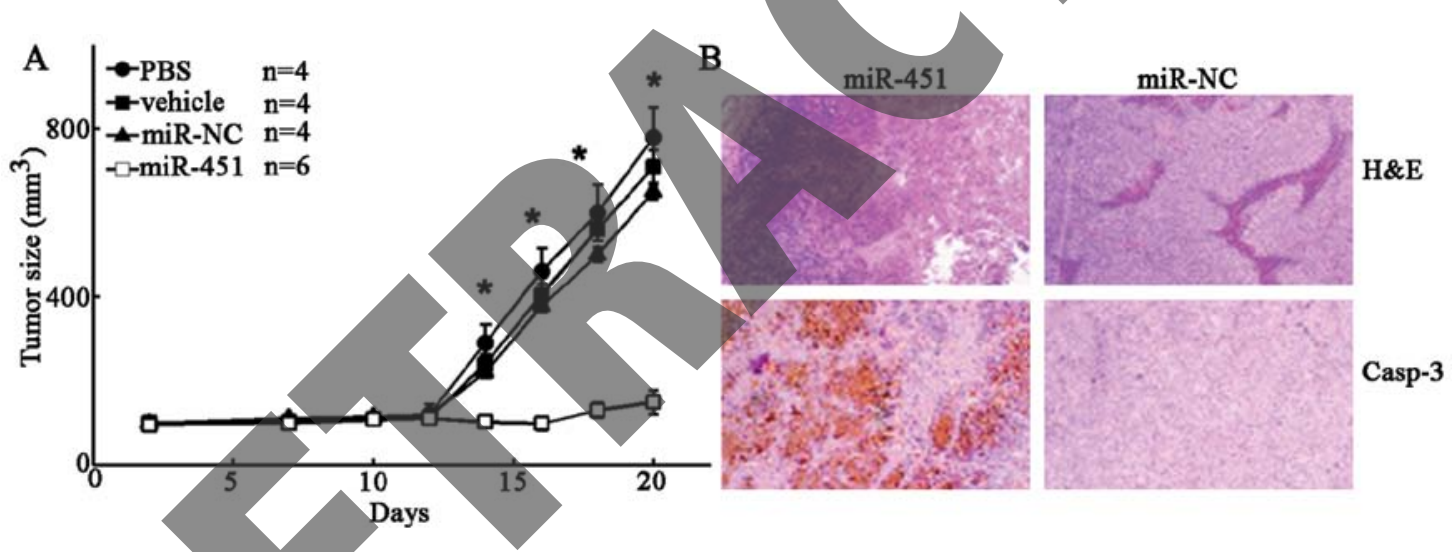

Figure 3. Local delivery of formulated miR-451 inhibits lung cancer tumor growth. (A) Effects of formulated miR-451 in H460 tumors by intratumoral (i.t.) injection. Subcutaneous H460 tumor xenografts were treated on days 12, 14 and 16 with $200 \mu \mathrm{g}$ of miR- 451 or miR-NC. Bars, standard deviation (SD). ${ }^{*} \mathrm{P}<0.05$ (Student's t-test, two-tailed). (B) Histologies directed against caspase-3 (Casp-3) in H460 tumors. Magnification, x100.

the growth inhibition was not restricted to cell lines with reduced endogenous miR-451 expression (e.g., H266 cells) (Figs. 1A and 2A). Colony formation assay used in A549 cells also showed consistent results (Fig. 2B). We then transfected miR-451 into H226 cells to investigate the long-term effect of miR-451 in proliferating cells. Cell counts were taken during the experiments and extrapolated to calculate final numbers. Cells with miR-NC transfection exhibited normal exponential growth (Fig. 2C). By contrast, miR-451 significantly inhibited H226 cell growth (Fig. 2C). The cells with miR-451 transfection showed signs of stress by loss of normal shape, followed by detachment (Fig. 2D). Thus, miR-451 exerts an anti-proliferative effect on various lung cancer cells.

Intratumoral delivery of miR-451 retards the growth of lung tumors in mice. We aimed to verify whether administration of
miR-451 was able to block tumor cell growth in mice. miRNAs were initially degraded in biofluids and the miRNAs were formulated in lipid-based delivery vehicle as mentioned in Materials and methods. We first evaluated the effect of i.t. injection using $100 \mu \mathrm{g}$ of oligos (i.e., an evidently growth-inhibitory concentration). H460 cells, which formed rapidly growing tumor xenografts within 3-4 weeks were grafted s.c. into the lower back of NOD/SCID mice until observable tumors were formed. On day 12 after xenograft implantation, a subgroup of mice received i.t. injection of formulated miR-451. The local injection of miR-451 was repeated at 14, 16 and 18 days post-implantation. The control group was i.t. injected with phosphate-buffered saline (PBS), vehicle only or vehicle with miR-NC. The results showed that tumor growth with miR-NC, vehicle only or PBS was not affected significantly (Fig. 3A). However, i.t. injection of miR-451 strongly blocked the growth 


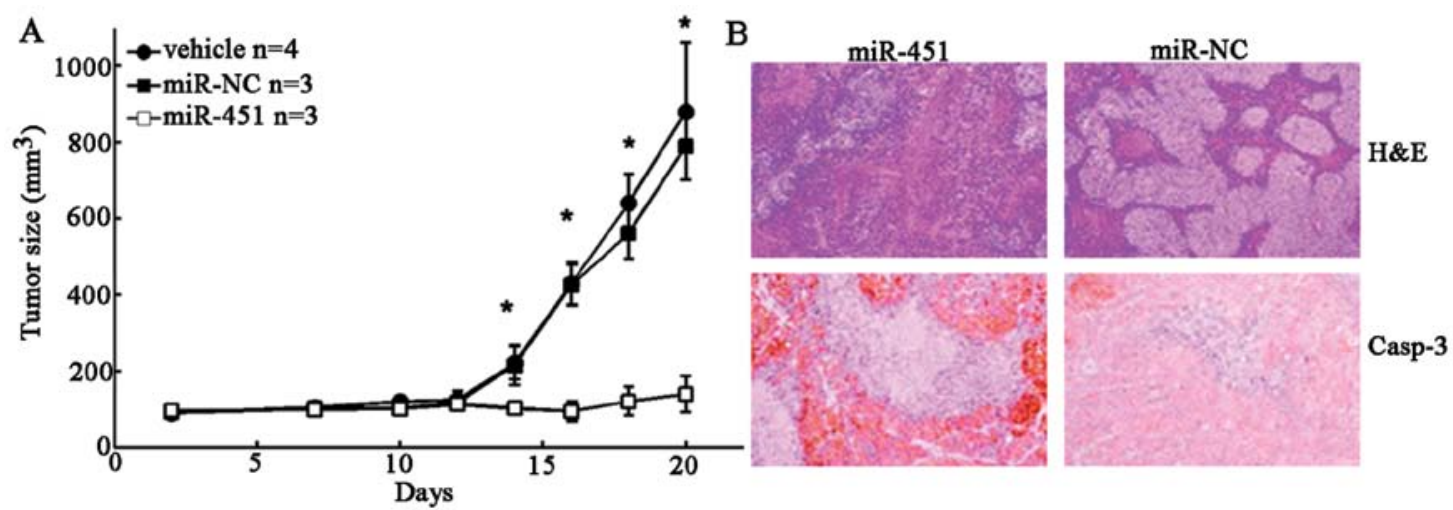

Figure 4. Intravenous delivery of formulated miR-451 blocks lung cancer tumor growth in mice. (A) Subcutaneous H460 tumor xenografts were treated on days 12,14 and 16 with $200 \mu \mathrm{g}$ of miR-451 or miR-NC. Bars, standard deviation (SD). ${ }^{*} \mathrm{P}<0.05$. miR-451 vs. miR-NC (Student's t-test, two-tailed). (B) Histologies directed against caspase-3 (Casp-3) in H460 tumors. Magnification, x100.
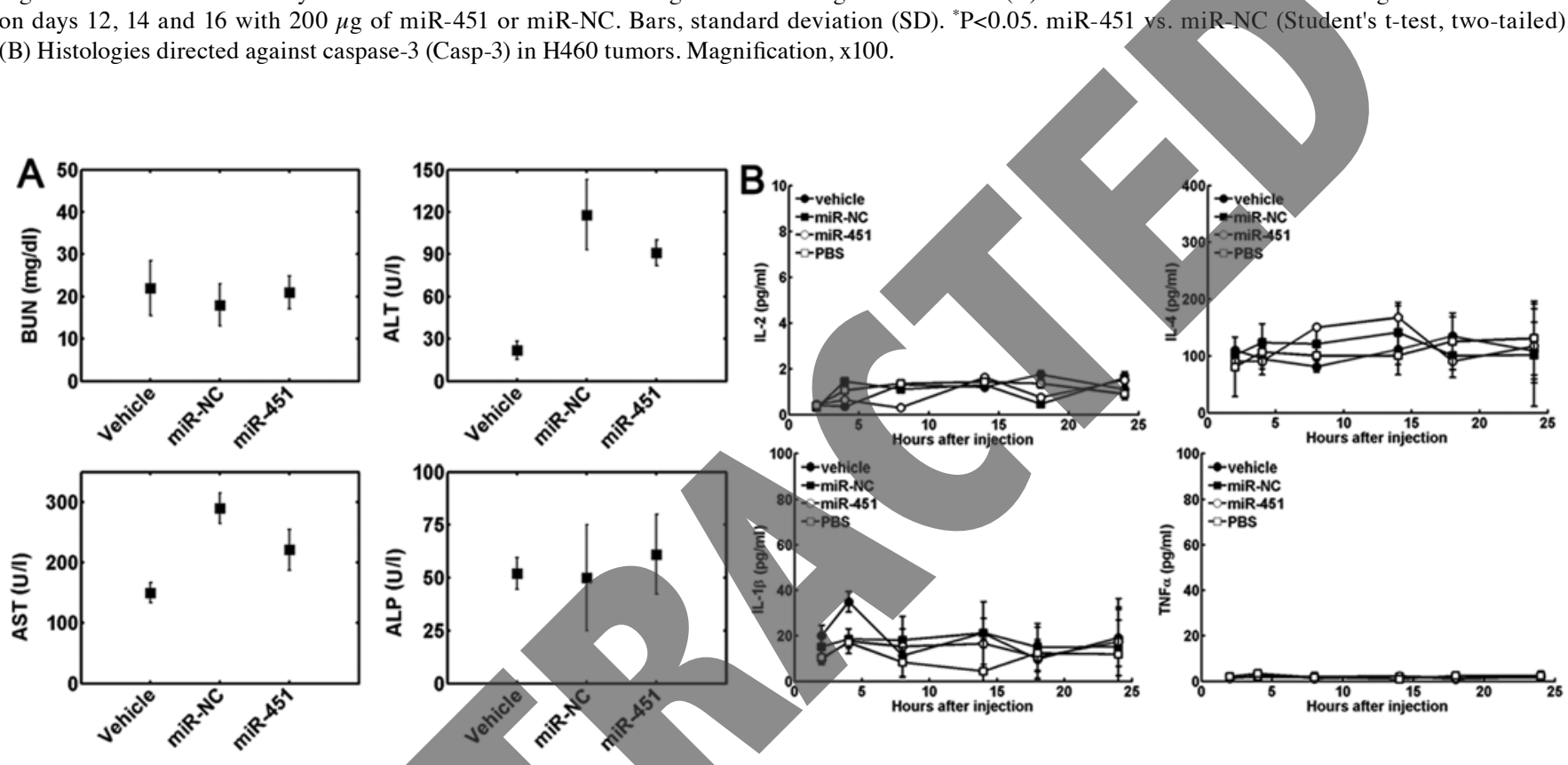

Figure 5. Blood chemistry and cytokine levels to systemic delivery of miR-451. (A) Serum levels of blood urea nitrogen (BUN), alkaline phosphatase (ALP), alanine aminotransferase (ALT), aspartate aminotransferase (AST). Points were average for four animals. Bars, standard deviation (SD). (B) Serum cytokine dynamics (IL-2, IL-4, IL-1 $\beta$ and TNF- $\alpha$ ) in BALB/c mice 2-h after intravenous (i.v.) injection of the same dose of miR-451, miR-NC, vehicle and phosphate-buffered saline (PBS). Points were average of four animals per group. Bars, standard deviation (SD).

of tumor xenograft (Fig. 3A). Histologic analysis further revealed that miR-451 transfected cells harbored large areas of debris. A substantial increase in caspase-3 was observed suggesting that miR-451 may inhibit proliferation by triggering apoptosis (Fig. 3B). These results suggested that local administration of miR-451 may exert a growth inhibitory effect on tumor cells.

Systemic delivery of formulated miR-451 inhibits the proliferation of lung tumors in vivo. To investigate the in vivo delivery effects, we repeated the experimental designs using H460 xenograft. Mice with observable tumors were injected with formulated miR-451, miR-NC or vehicle only. The difference identified was that the injection was administered i.v. at the tail vein. Each dose contained $100 \mu \mathrm{g}$ of formulated oligos. Consecutive delivery of miR-451 substantially inhibited tumor growth (Fig. 4A). Caspase-3 upregulation was also evident compared with the controls (Fig. 4B). The local and systemic delivery of miR-451 showed consistent results.
Systemic delivery of miR-451 does not significantly alter the blood chemistries or normal inflammatory responses. Safety in miRNA delivery is an important issue to be evaluated. Therefore, we examined the serum levels of alanine aminotransferase (ALT), aspartate aminotransferase (AST), blood urea nitrogen (BUN) and alkaline phosphatase (ALP) in mice treated with repeated vehicle, miR-NC and miR-451. The results suggested that the blood chemistries were all within the normal ranges and living organisms were able to tolerate the miRNA treatment (Fig. 5A). We also measured the serum cytokine levels of in mice. We measured the cytokine levels from 2 to $24 \mathrm{~h}$ after i.v. injection of miR-451. No significant alterations in cytokine levels in miR-451, miR-NC, PBS or vehicle-treated mice were identified (Fig. 5B).

\section{Discussion}

miRNAs are a class of small, non-coding RNAs that regulate gene expression by targeting mRNAs for translational repres- 
sion or degradation. Accumulating evidence suggests that the deregulation of miRNAs has been frequently observed in tumor tissues. These miRNAs have regulatory roles in the pathogenesis of cancer $(14,15)$. However, direct administration of formulated miRNAs is less reported.

The results of this study provide evidence that miR-451 can be locally or systemically administered and harbors a therapeutic effect. By introducing tumor-suppressor miRNA particularly for those that exhibited mutations in critical antitumor genes, miRNA therapy may reactivate the anticancer signaling pathways and may restore normal cell function. Formulated miR-451 injection can effectively suppress tumor growth as was evident by the elevated caspase-3 expression and debris. These results are consistent with a previous study showing that upregulation of miR-451 enhanced caspase-3dependent apoptosis of A549 cells by inactivating the Akt signaling pathway which induced the reverse of the Bcl-2/Bax ratio (6). The strong oncogenic potential may be explained partially by the fact that miRNAs target various pathway components (16). Simultaneous targeting multiple oncogenic pathways may the key to cancer therapy. Other examples that support the conceptual framework of miRNA replacement therapy are supplied by miR-34a, let-7, miR-16 and miR-26a, all of which are tumor-suppressor miRNAs and may retard tumor growth in tumor-bearing mouse models (17-20).

However, whether miRNA administration is safe remains an issue of active research. In the present study, we reported that miR-451 administration proved to be a safe strategy as cells tolerated miR-451 injection. Blood chemistries and inflammatory responses were normal irrespective of miR-451 administration. Therefore, systemic delivery may be applicable. miR-451 can be transcriptionally repressed by STAT3 (10). Notably, STAT3 transcription factors are detected at high levels or constitutively activated in a number of cancer cells $(10,21,22)$. Therefore, extrinsic administration of miR-451 may overcome the oncogenic potential in various tumor cells probably with STAT3 deregulation.

In conclusion, this study provides a promising strategy for lung cancer treatment via miR-451 replacement therapy. The synthetic miRNA delivery can be performed in controllable conditions and can overcome various technical constraints in traditional gene therapy. However, studies are required to demonstrate the efficacy and safety in other species, particularly humans.

\section{References}

1. Lewis BP, Burge CB and Bartel DP: Conserved seed pairing, often flanked by adenosines, indicates that thousands of human genes are microRNA targets. Cell 120: 15-20, 2005.

2. Fabbri M: miRNAs as molecular biomarkers of cancer. Expert Rev Mol Diagn 10: 435-444, 2010.
3. Baranwal S and Alahari SK: miRNA control of tumor cell invasion and metastasis. Int J Cancer 126: 1283-1290, 2010.

4. Wang R, Wang ZX, Yang JS, Pan X, De W and Chen LB: MicroRNA-451 functions as a tumor suppressor in human non-small cell lung cancer by targeting ras-related protein 14 (RAB14). Oncogene 30: 2644-2658, 2011.

5. Kovalchuk O, Filkowski J, Meservy J, et al: Involvement of microRNA-451 in resistance of the MCF-7 breast cancer cells to chemotherapeutic drug doxorubicin. Mol Cancer Ther 7: 2152-2159, 2008.

6. Bian HB, Pan X, Yang JS, Wang ZX and De W: Upregulation of microRNA-451 increases cisplatin sensitivity of non-small cell lung cancer cell line (A549). J Exp Clin Cancer Res 30: 20, 2011.

7. Hui AB, Lenarduzzi M, Krushel T, et al: Comprehensive MicroR NA profiling for head and neck squamous cell carcinomas. Clin Cancer Res 16: 1129-1139, 2010.

8. Bitarte N, Bandres E, Boni V, et al: MicroRNA-451 is involved in the self-renewal, tumorigenicity, and chemoresistance of colorectal cancer stem cells. Stem Cells 29: 1661-1671, 2011.

9. Kang Y, He W, Tulley S, et al: Breast cancer bone metastasis mediated by the Smad tumor suppressor pathway. Proc Natl Acad Sci USA 102: 13909-13914, 2005.

10. Rozovski U, Calin GA, Setoyama T, et al: Signal transducer and activator of transcription (STAT)-3 regulates microRNA gene expression in chronic lymphocytic leukemia cells. Mol Cancer 12: 50,2013

11. Bandres E, Bitarte N, Arias F, et al: mícroRNA-451 regulates macrophage migration inhibitory factor production and proliferation of gastrointestinal cancer cells. Clin Cancer Res 15: 2281-2290, 2009

12. Wang T, Zang WQ, Li M, Wang N, Zheng YL and Zhao GQ: Effect of miR-451 on the biological behavior of the esophageal carcinoma cell line EC9706. Dig Dis Sci 58: 706-714, 2013.

13. Zhang Y, Liu D, Chen X, et al: Secreted monocytic miR-150 enhances targeted endothelial cell migration. Mol Cell 39: 133-144, 2010.

14. Dykxhoorn DM: MicroRNAs and metastasis: little RNAs go a long way. Cancer Res 70: 6401-6406, 2010.

5. Hummel R, Hussey DJ and Haier J: MicroRNAs: predictors and modifiers of chemo- and radiotherapy in different tumour types. Eur JCancer 46: 298-311, 2010.

16. Esquela-Kerscher A and Slack FJ: Oncomirs - microRNAs with a role in cancer. Nat Rev Cancer 6: 259-269, 2006.

. Esquela-Kerscher A, Trang P, Wiggins JF, et al: The let-7 microRNA reduces tumor growth in mouse models of lung cancer. Cell Cycle 7: 759-764, 2008.

18. Kota J, Chivukula RR, O'Donnell KA, et al: Therapeutic microRNA delivery suppresses tumorigenesis in a murine liver cancer model. Cell 137: 1005-1017, 2009.

19. Takeshita F, Patrawala L, Osaki M, et al: Systemic delivery of synthetic microRNA-16 inhibits the growth of metastatic prostate tumors via downregulation of multiple cell-cycle genes. Mol Ther 18: 181-187, 2010.

20. Wiggins JF, Ruffino L, Kelnar K, et al: Development of a lung cancer therapeutic based on the tumor suppressor microRNA-34. Cancer Res 70: 5923-5930, 2010.

21. Yu H, Kortylewski M and Pardoll D: Crosstalk between cancer and immune cells: role of STAT3 in the tumour microenvironment. Nat Rev Immunol 7: 41-51, 2007.

22. Garner JM, Fan M, Yang CH, et al: Constitutive activation of signal transducer and activator of transcription 3 (STAT3) and nuclear factor $\mathrm{\kappa B}$ signaling in glioblastoma cancer stem cells regulates the Notch pathway. J Biol Chem 288: 26167-26176, 2013. 